\title{
Extensive adipocytic maturation can be seen in myxoid liposarcomas treated with neoadjuvant doxorubicin and ifosfamide and pre-operative radiation therapy
}

Wei-Lien Wang ${ }^{1}$, Daniela Katz ${ }^{2}$, Dejka M Araujo ${ }^{3}$, Vinod Ravi ${ }^{3}$, Joseph A Ludwig ${ }^{3}$, Jonathan C Trent ${ }^{4}$, Shreyaskumar R Patel ${ }^{3}$, Patrick P Lin ${ }^{5}$, Ashleigh Guadagnolo ${ }^{6}$, Dolores Lòpez-Terrada', Angelo Paola dei Tos ${ }^{8}$, Valerie O Lewis ${ }^{5}$, Dina Lev ${ }^{9,10}$, Raphael E Pollock ${ }^{10,11}$, Gunar K Zagars ${ }^{6}$, Robert S Benjamin ${ }^{3}$, John E Madewell ${ }^{12}$ and Alexander J Lazar ${ }^{1,10^{*}}$

\footnotetext{
Abstract

Background: Trabectedin and thioglitazones have been documented to induce adipocytic maturation in myxoid liposarcoma; we have noted this in our experience as well. Intriguingly, we have also encountered this same phenomenon in myxoid liposarcomas exposed to various combinations of neoadjuvant doxorubicin and ifosfamide systemic chemotherapy with preoperative radiation, where the pathological effects have been less characterized. We examined the histological changes, including adipocytic maturation, associated with this treatment in patients with myxoid liposarcoma and evaluated for prognostic significance.

Methods: Twenty-two patients were identified with histologically confirmed myxoid liposarcomas (9 with variable hypercellular areas) who were treated with neoadjuvant doxorubicin $(75-90 \mathrm{mg} / \mathrm{m} 2 /$ continous infusion over $72 \mathrm{~h}$ every 3 week) and ifosfamide ( $2.5 \mathrm{~g} / \mathrm{m} 2$ daily $\times 4$ every 3 weeks) for 4-6 cycles. Twenty-one patients received pre-operative radiation including 5 with concurrent gemcitabine. Pre- and post-treatment MRI studies were compared for changes in tumor area, fat content and contrast uptake, with the latter two estimated as: none, $<25 \%, 25-49 \%$ and $>50 \%$. Post-treatment specimens were evaluated for hyalinization, necrosis and adipocytic maturation. Clinical follow-up was obtained.

(Continued on next page)
}

\footnotetext{
* Correspondence: alazar@mdanderson.org

${ }^{1}$ Department of Pathology, The University of Texas MD Anderson Cancer Center, 1515 Holcombe Blvd Unit 085, Houston, TX 77030, USA

${ }^{10}$ Sarcoma Research Center, The University of Texas MD Anderson Cancer

Center, 1515 Holcombe Blvd Unit 085, Houston, TX 77030, USA

Full list of author information is available at the end of the article
} 
(Continued from previous page)

Results: Median age was 45 (26-72) years with a median tumor size of $11(2-18) \mathrm{cm}$. All occurred in the lower extremities except for one case in the neck. As is common in myxoid liposarcoma, all had extensive treatment changes ( $>90 \%)$ with extensive hyalinization $(n=16 ;>90 \%)$ or prominent adipocytic maturation $(n=6 ;>50 \%)$ including 2 cases composed almost entirely of mature-appearing adipose tissue. Variable necrosis was identified (5-30\%). MRI revealed a decrease in tumor area in all but one tumor (median, 65\%), an increase in fat content in 7 tumors $(n=2,>50 \% ; n=2,25-50 \% ; n=3,<25 \%)$, and a decrease in contrast enhancement in most tumors ( $n=5,>50 \% ; n=9,25-49 \% ; n=7,<25 \%)$. Median follow-up was 57 (12-96) months with 17 alive with no disease/metastases, 3 alive with disease and 2 dead of disease. Six patients developed metastases with median interval of 26 (22-51) months post resection. Four of 6 tumors with increased adipocytic maturation $>50 \%$ on histology had increased fat detected by MRI (>25\%). All 6 are alive but 2 developed metastases. In the remaining patients, 4 developed metastases with 14 alive and 2 dead of disease.

Conclusion: Myxoid liposarcoma exposed to neoadjuvant doxorubicin and ifosfamide and pre-operative radiation can have prominent adipocytic maturation similar to trabectedin treatment. Myxoid liposarcomas exhibit extensive treatment changes with prominent hyalinization being the most common histological change. Despite this, patients develop metastases regardless of adipocytic maturation. While of unclear significance, no patient with fatty maturation died of disease.

Keywords: Adipocytic maturation, Myxoid liposarcoma, Treatment

\section{Background}

Myxoid liposarcoma is the second most common malignant lipomatous tumor and preferentially affects ages younger than most other liposarcomas (young adults), and typically occurs in the lower extremities. Classically, these tumors are characterized by monotonous small short spindle cells in myxoid stroma with abundant thin-walled capillaries. Scattered lipoblasts, typically univacuolar, are variably present sometimes accompanied by variable amounts of more mature-appearing adipocytes $[1,2]$. Despite their banal histological appearance, metastases are common, and often to peculiar sites such as to the retroperitoneum and bone $[3,4]$. Tumors with increased hypercellular areas composed of enlarged, more round tumor cells without intervening myxoid stroma have an increased risk for metastases and poor prognosis [2-6]. Those with a significant proportion of round cell change are termed round cell liposarcoma and considered to be a higher grade variant of myxoid liposarcoma [7].

The vast majority of myxoid liposarcomas are characterized by a recurrent translocation involving $\mathrm{t}(12 ; 16)$ (q13;p11) fusing the 3 -end promoter region of FUS/TLS (16p11) with the $5^{\prime}$-end of the CHOP/DDIT3 locus (12q13) [8-14]. The resulting fusion protein is believed to play a critical role in tumorigenesis, blocking the PPAR $\gamma$ pathway which promotes adipocytic differentiation or maturation, offering insight into the primitive preadipocytic histological appearance of this tumor $[15,16]$. In rare situations, the alternative $\mathrm{t}(12 ; 22)$ (q13;p11) resulting in a EWSR1-CHOP/DDIT3 fusion transcript is present $[9,17-20]$. Other genetic mutations including involvement of the p53, RET, MET, and PI-3 kinase pathways, have also been implicated in myxoid liposarcomas [6,15,16,21-23].

ET-743 (also known as trabectedin) is an alkaloid compound discovered from the sea squirt Ecteinascidia turbinata and found to show efficacy in the treatment of sarcoma patients including those with myxoid liposarcoma [24-27]. While the mechanism of action is not completely known, trabectedin may block transcription factor binding to the fusion gene FUS-CHOP/DDIT3 [15]. It has also been well documented to induce differentiation in myxoid liposarcoma with prominent adipocytic maturation (Figure 1). In addition, PPAR $\gamma$ agonists, thioglitazones, have been reported to produce similar histological effects in

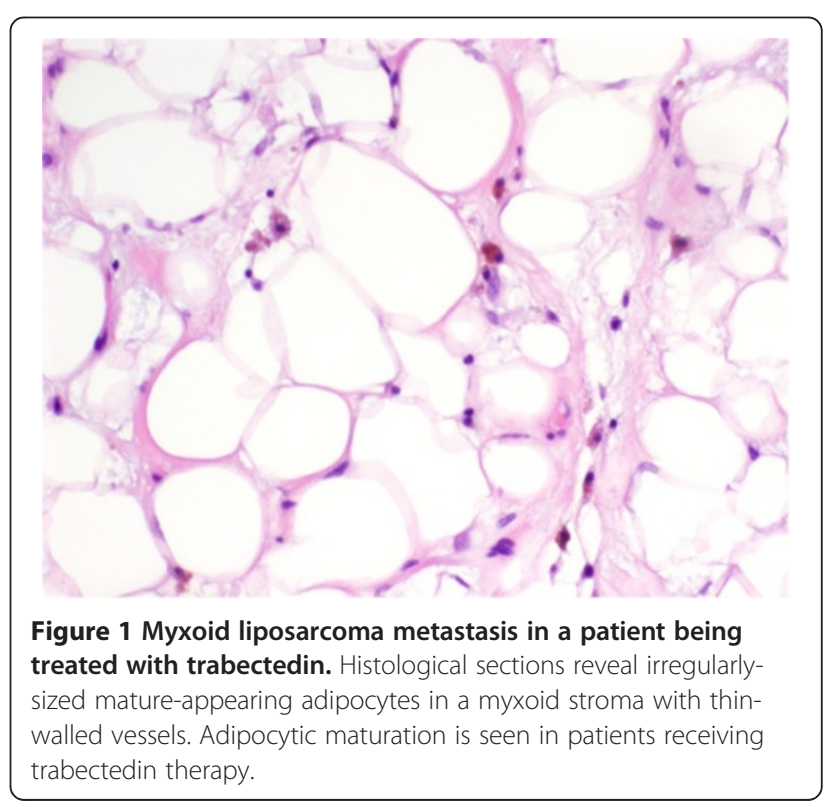


myxoid liposarcomas [28]. These effects have been purported to be characteristic and perhaps specific and thus suggesting a particular mechanism of action leading to the release of a blockade of adipocytic maturation in the tumor.

We have encountered this phenomenon of adipocytic maturation in myxoid liposarcomas exposed to neoadjuvant doxorubicin and ifosfamide and pre-operative radiation, where the pathological effects have been less characterized. We examined the histological changes, including adipocytic maturation, associated with this treatment in patients with myxoid liposarcoma and evaluated for any prognostic significance of this feature.

\section{Methods}

Under institutional board review approval, twenty-two patients were identified between 2000 and 2009 who had histologically confirmed (WLW, AJL) myxoid liposarcoma on pre-treatment biopsy and were not previously treated with chemotherapy. Nine of these patients had variable hypercellular/round cell change. Patients were treated with neoadjuvant doxorubicin $(75-90 \mathrm{mg} / \mathrm{m} 2 /$ continous infusion over 72 hours every three weeks) and ifosfamide $(2.5 \mathrm{~g} / \mathrm{m} 2$ over three hours for four days, every three weeks) for 4-6 cycles. Twenty-one patients also received preoperative radiation including 5 with concurrent gemcitabine. Pre- and post-treatment MRI studies were compared (JM) for changes in tumor area, fat content and contrast uptake, with the latter two estimated as: none, $<25 \%, 25-4.9 \%$ and $>50 \%$. Post-treatment specimens were compared with pre-treatment biopsies and evaluated for percentage of hyalinization, necrosis and adipocytic maturation (WLW, AJL). Clinical followup was obtained. Fluorescence in situ hybridization (FISH) on a selected post-treatment case for $12 \mathrm{q} 13$ to detect CHOP/DDIT3 rearrangement was performed using the LSI FUS dual-color, break-apart probe (Abbott Molecular/Vysis, Des Plaines, IL, USA) according to the manufacturer's recommendations as previously described [14].

\section{Results}

Clinical, pathological and radiological findings are summarized in Table 1. The median age was 45 (26-72) years with a median size of $11(2-18) \mathrm{cm}$. All occurred in the lower extremities except for one case in the neck.

All developed extensive post-treatment changes $(>90 \%)$ with extensive hyalinization $(>90 \%)$ being the most common change seen (16/22 patients, 72\%) (Figure 2). Focal residual areas of characteristic vascular pattern were often identified. Prominent fatty maturation $(>50 \%)$ was seen in 6/22 (27\%) cases including 2 cases composed almost entirely of mature-appearing fat (Figure 3). Both cases received radiation therapy; one also received gemcitabine. One tumor with extensive post-treatment fatty maturation was tested and found to retain the rearrangement in CHOP/DDIT3 verifying that the mature adipocytes arose from the tumor cells. Variable necrosis was also identified (5-30\%).

MRI revealed a decrease in tumor area in all but one tumor (median decrease in size was 65\%). A decrease in contrast enhancement was found in most tumors

Table 1 Summary of clinical, pathological and imaging factors

\begin{tabular}{ll}
\hline Median age (range) years & $\mathbf{4 5}(\mathbf{2 6 - 7 2 )}$ \\
\hline Sex M:F & $13: 9$ \\
Location (n, \%) & \\
Thigh & $15(68 \%)$ \\
Calf & $6(27 \%)$ \\
Neck & $1(4.5 \%)$ \\
Round Cell Component (n,range of \%) & $\mathrm{n}=9(5-30 \%)$
\end{tabular}

\section{Follow-up}

Median, range

Alive and Well (median, range)

Alive with Disease (range)

Dead of Disease (range)

Metastases (median, range)

57 mos (12-96 mos)

$\mathrm{n}=17$ (35 mos, 12-96 mos)

$n=3(61,67,72$ mos $)$

$\mathrm{n}=2(82,16 \mathrm{mos})$

$n=6(26$,

22-51 mos)

Post-Treatment Pathological Findings

Median Size (range)

$11(2-18) \mathrm{cm}$

Tumor with Treatment Changes

(median \%, range)

95\% (90-95\%)

$90 \%(10-95 \%)$

Hyalinization (median \%, range)

Fat Maturation

Median (range)\%

$10 \%(5-90 \%)$

Prominent $>50 \%$ (n)

$\mathrm{n}=6$ with $2>90 \%$

5\% (5-10\%)

Radiological Changes

Decrease in Tumor Size

(median \% decrease, range)

Fat Amount Change

( $n, \%$ of cases with changes)

Decrease $<25 \%$

$1(4.5 \%)$

No change

$14(63.6 \%)$

Increase $<25 \%$

$3(13.6 \%)$

Increase 25-49\%

$2(9.1 \%)$

Increase $>50 \%$

$2(9.1 \%)$

\section{Contrast Media Change}

( $n, \%$ of cases with changes)

Increase $<25 \%$

$1(4.5 \%)$

Decrease $<25 \%$

$7(31.8 \%)$

Decrease 25-49\%

9 (40.9\%)

Decrease $>50 \%$

$5(22.7 \%)$

* One specimen had an increase in size (17\%). 


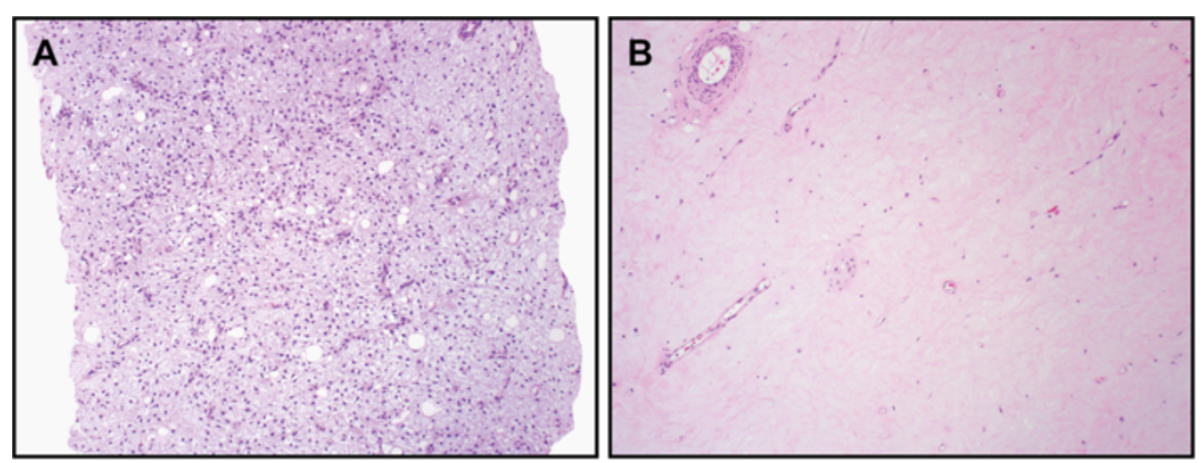

Figure 2 (A) Pre-treatment biopsy reveals characteristic bland spindle cells in myxoid stroma with branching thin walled vessels. (B) Post treatment specimen reveals extensive hyalinization that is essentially acellular. This was the most common pattern seen in treated tumors.

$(\mathrm{n}=5,>50 \% ; \mathrm{n}=9,25-49 \% ; \mathrm{n}=7,<25 \%$ ) (Figure 4 ), histologically corresponding to increased hyalinization, decreased cellularity and decreased vasculature. Seven tumors developed an increase in a register characteristic of mature adipose tissue $(n=2,>50 \%$; $n=2,25-49 \%$; $\mathrm{n}=3,<25 \%$ ) (Figure 5). Four of 6 tumors with histological adipocytic maturation $>50 \%$ also had increased adipose signal detected by MRI ( $>25 \%)$.

Median follow-up was 57 (12-96) months with 17 patients alive with no disease/metastases, 3 patients alive with disease and 2 patients dead of disease. Six patients developed metastases with a median interval of 26 (22-51) months post resection. All 6 patients with adipocytic maturation are alive although 2 developed metastases.

\section{Conclusions}

Myxoid liposarcomas treated with neoadjuvant doxorubicin and ifosfamide and pre-operative radiation consistently develop extensive treatment effect that can

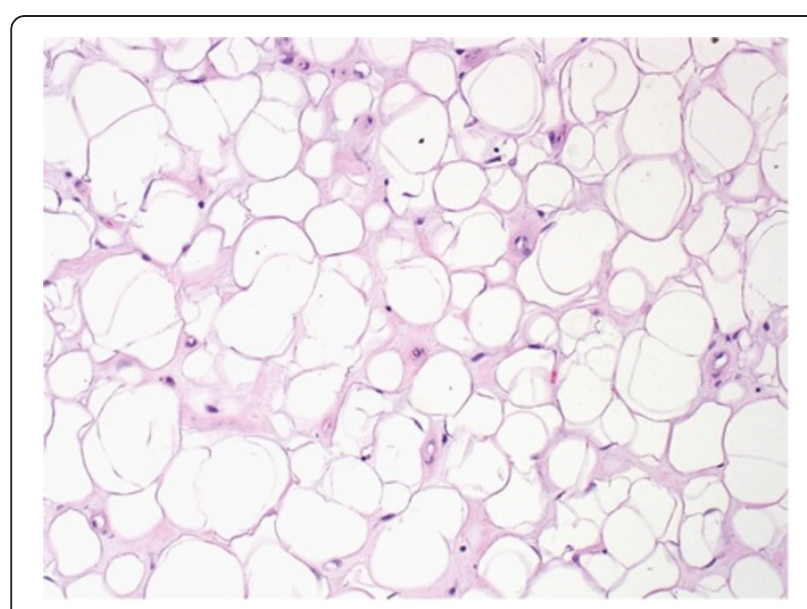

Figure 3 Histological example of extensive fatty maturation with variable sized adipocytes. Extensive fatty maturation was seen in one-third of cases; two of which had adipocytic maturation that was greater than $90 \%$. include adipocytic maturation as demonstrated on MRI imaging and confirmed histologically at resection. In our series, the most common treatment change was extensive hyalinization $(16 / 22,72 \%)$ with decreased vasculature as seen in other studies [29]. In addition, approximately one-third of our patients also developed prominent adipocytic maturation, comprising more than $50 \%$ of the treated tumor. Two tumors had extensive adipocytic differentiation giving a misleading appearance of a welldifferentiated liposarcoma or even a benign lipoma. Fortunately, most tumors also harbored areas of hyalinization, characteristic of treated myxoid liposarcoma. They also lacked the fibrous bands with scattered pleomorphic cells seen in most well-differentiated liposarcomas. Molecular testing, either for rearrangements involving CHOP/DDIT3 by fluorescence in-situ hybridization assay or reverse polymerase chain reaction for the specific fusion transcripts, can also be helpful in confirming the diagnosis of myxoid liposarcoma [14,30]. In our study, rearrangement was still identified in tumors with prominent fatty differentiation by fluorescence in-situ hybridization assay. We chose this modality since it allows one to determine the cellular component of the tissue showing the re-arrangement. Well-differentiated liposarcomas would lack this rearrangement and would instead demonstrate amplification of the 12q13-15 region [31].

The phenomenon of adipocytic maturation is comparable to myxoid liposarcomas in patients treated with trabectedin and the thioglitazone family of PPAR $\gamma$ agonists. In 2007, Grosso and colleagues described the development of "mature lipoblast-featuring cells" and "clear adipocytic differentiation" in myxoid liposarcomas treated with trabectedin [24]. Gronchi and colleagues reported maturation to lipoblasts, decreased cellularity and vascularity in the tumors of 16 of 23 patients who received neoadjuvant trabectedin for advanced localized myxoid liposarcomas [32]. Forni and colleagues saw similar findings in myxoid liposarcoma cell lines and two patients treated with trabectedin [33]. Similarly, Demetri and 

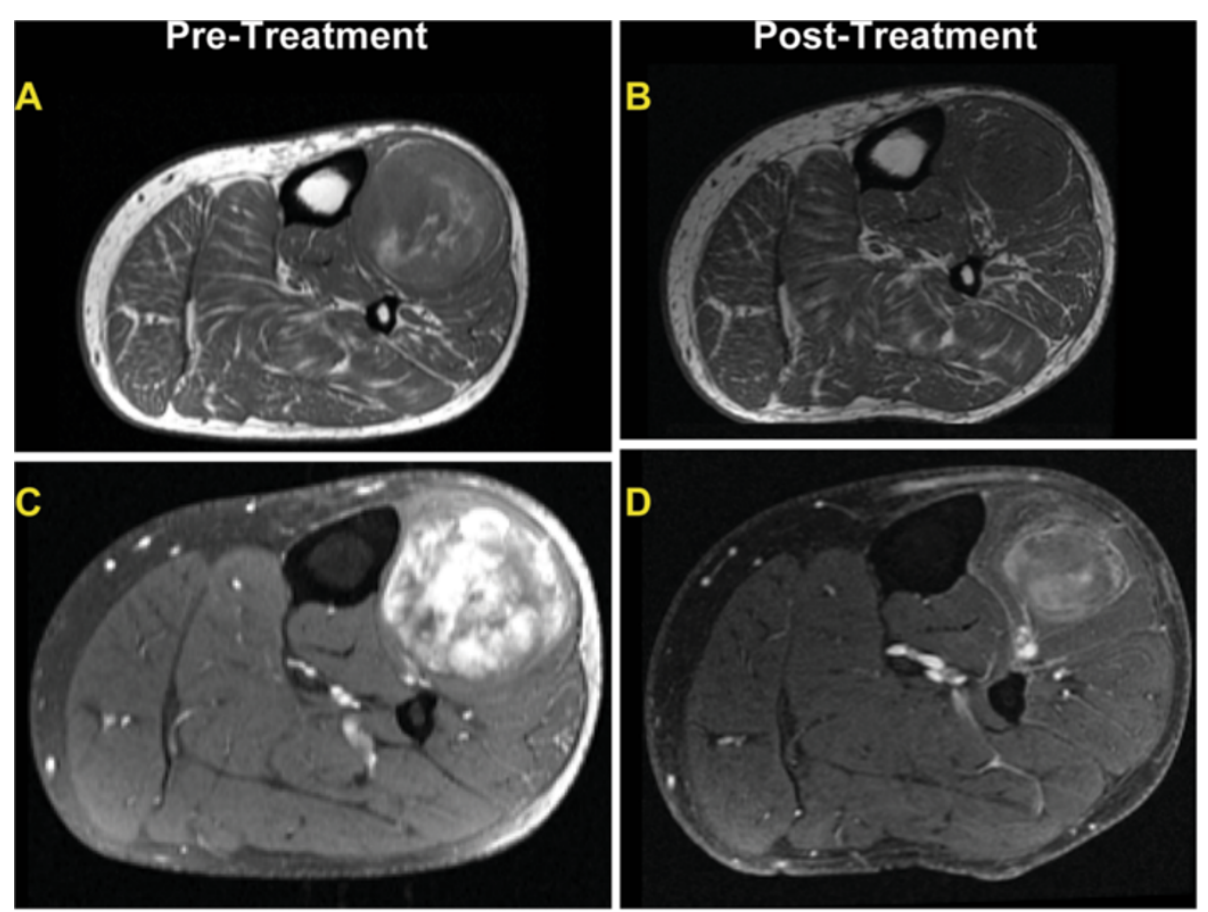

Figure 4 Example of MRI findings seen in the majority of myxoid liposarcomas undergoing treatment in our series. $T 1$ weighted axial image of the calf with mass (A) pre-treatment and (B) post treatment showing a reduction in tumor size and decreased adipocytic content. T1 weighted post contrast media with fat saturation with $(\mathbf{C})$ pre-treatment demonstrating an extensive heterogeneous enhancement, while (D) post treatment shows decreased tumor size and decreased enhancement greater than 50\%. These findings correspond to increased hyalinization and decreased vascularity histologically.

colleagues also saw adipocytic differentiation as early as six weeks in tumors from three patients treated with troglitazone, a PPARy agonist. These changes coincided with an increased expression in mRNA associated with adipocytic differentiation including AP2, adipsin and PPAR $\gamma$. Mild increase in adipose density signaling was also seen in these tumors on MRI imaging [28]. In our series, extensive treatment changes, predominantly hyalinization with decreased cellularity and vascularity, was seen in the tumors of our patients treated with doxorubicin and ifosfamide with pre-operative radiation therapy. However, adipocytic maturation was also seen in our tumors, histologically indistinguishable to what has been reported previously reported by others and also seen by us in myxoid liposarcoma tumors treated with trabectedin (WLW, AJL, PT). In most cases, MRI imaging correlated with evolution of treatment changes seen in the resection specimen. Decreased vascularity by contrast media enhanced imaging was seen in the majority of cases with extensive hyalinization and subsequent decrease of the thin-walled vessels in these areas. In addition, all four cases that had an increase of fat $(>25 \%)$ by MRI also showed prominent adipocytic maturation on histological resection.

Myxoid liposarcoma can rarely have areas with mature-appearing adipocytes as well. Evans reported 29 cases of myxoid liposarcoma, some of which had areas that were reported to resemble mature, non-neoplastic adult adipose tissue [1]. In a more recent study by Fritchie and colleagues, 10 of 46 (22\%) myxoid liposarcomas had a mature fat appearing lipomatous component that composed $5-79 \%$ of the tumor. Full information regarding treatment was not provided in either study. Some degree of stromal hyalinization was also observed in seven cases in the Fritchie study, six of which were known to be untreated. However, this was only focal, composing only $1-9 \%$ of these tumors, in contrast to our treated cases which had extensive hyalinization [7]. It is possible that some changes could have been present before treatment and not sampled in the pre-treatment biopsy, emphasizing the importance of comparing pre- and post-treatment imaging studies. One case with histological evidence of adipocytic maturation revealed no change in adipocytic content by MRI and adipocytic maturation was noted in the pretreatment biopsy. However, in our experience (WLW, AJL) extensive fatty maturation and hyalinization are uncommon in untreated myxoid liposarcomas. The remaining pretreatment biopsies lacked these changes and developed increased fat on MRI studies between pre- and post treatment tumors, which corresponded histologically to increased fatty maturation, increased hyalinization and decrease vascularity on the resection specimen. 

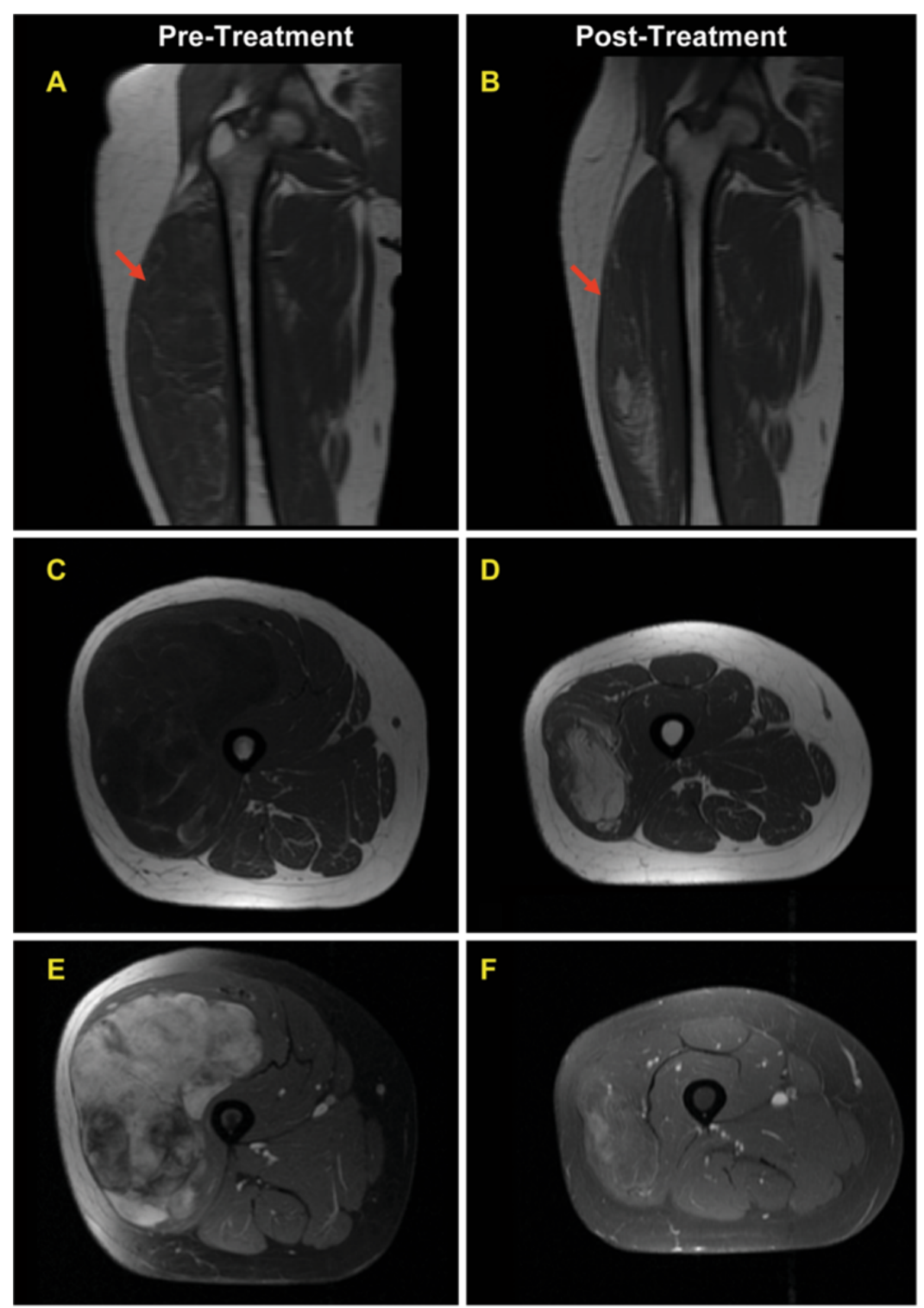

Figure 5 MRI findings in a case of myxoid liposarcoma which developed extensive adipocytic maturation during treatment. T1

weighted coronal right thigh (A) pre-treatment and (B) post treatment demonstrating a marked decrease in tumor size. T1 weighted axial image of right thigh (C) pre-treatment and (D) post treatment demonstrate an increase in signal characteristic of mature adipose tissue content ( $>50 \%)$ with reduction in size. T1 weighted axial with contrast media and fat saturation (E) pre-treatment showing large mass with extensive vascular enhancement. (F) Post treatment scans show a reduction in size of mass and a decrease in vascular enhancement greater than $50 \%$.

The significance of adipocytic maturation is unclear. No patients with extensive adipocytic maturation died of disease, but patients did develop metastases regardless of adipocytic maturation. However, our series is small and additional larger studies are necessary. The mechanism of fatty maturation when exposed to doxorubicin and ifosfamide and radiation therapy is also not known. Doxorubicin is an anthracycline and proposed to have many mechanisms of actions among which include intercalcating DNA, formation of free radicals, inhibition of topoisomerase II, prohibiting DNA and RNA unwinding, and induction of cell death [34]. Ifosfamide is a DNA 
alkylating agent which prohibits DNA replication and results in cell death [35]. Interestingly, doxorubicin at low concentrations has been reported to induce differentiation in leukemia and breast carcinoma cell lines [34,36,37]. In breast cell lines, differentiation corresponded to the reduction in c-myc expression and growth arrest in response to doxorubicin induced DNA damage [37]. To our knowledge, ifosfamide has not been reported to induce differentiation. Perhaps a similar non-specific response to DNA damage with doxorubicin can occur in myxoid liposarcomas. Other sarcomas have also been reported to differentiate in response to chemotherapy and radiotherapy including rhabdomyosarcoma [38]. Our study would support the notion that these changes can be a general response to effective treatment in myxoid liposarcoma and may not necessarily be specific to a treatment modality such as trabectedin. In this context, we should be cautious in interpreting potential insights into the unknown mechanism of action of trabectedin from this phenomenon.

The fusion transcript characteristic in the vast majority of myxoid liposarcomas is composed of FUS, which encodes for a RNA/DNA binding domain, and CHOP/DDIT3, which encodes for a transcription factor which dimerizes with CAAT/enhancer binding proteins $(\mathrm{C} / \mathrm{EBP})$ and negatively regulates genes involved in adipocytic differentiation including PPAR $\gamma$. Multiple fusion variants and types exist; however, none have been associated with increased adipogenesis [39]. Trabectedin binds to the minor groove of DNA and is theorized to sterically hinder the binding of the FUS-CHOP/DDIT3 fusion protein (probably mediated primarily by the CHOP/DDIT3-donated DNA binding domain) and thus allow adipocytic differentiation to continue by relieving repression. It could also effect the post transcriptional modification of the fusion transcript [15]. In addition, other mutations are known to exist including activation of the ERK/MAPK, PI-3-kinase/Akt pathways, alterations of p53, IGF1R and IGF2; some of these have been associated with round cell change and poor prognosis. $[16,22]$ None are known to correlate with adipocytic differentiation.

In summary, we report the histological and radiological findings seen in myxoid liposarcomas in patients treated with doxorubicin and ifosfamide and preoperative radiation therapy. The vast majority of these tumors demonstrated significant treatment effect in the form of hyalinization; however, adipocytic maturation, once thought to be exclusive to tumors treated with trabectedin and PPAR $\gamma$ agonists, were also seen in some of our cases. Pathologists and radiologists should be aware of this as it may cause diagnostic confusion if prior history is not available and in comparing histological treatment changes between trials.
Competing interests

The authors declare that they have no competing interests.

\section{Authors' contributions}

WLW reviewed the histological findings and wrote the publication; AJL reviewed the histological findings and is the senior author on this publication; PT reviewed selected histological findings; JM reviewed the radiological studies; $\mathrm{DL}$ edited the manuscript; $\mathrm{VOL}, \mathrm{PL}$, and $\mathrm{RP}$ surgically removed the specimens; $A G$ and $G Z$ performed the radiation treatment; $\mathrm{RB}, \mathrm{SP}, \mathrm{RV}, \mathrm{DA}$, and DK administered the chemotherapy, DLT performed the fluorescence in-situ hybridization. All authors read and approved the final manuscript.

\section{Acknowledgements}

We acknowledge Kim Vu for her expert assistance in the graphic arts. The Liddy Shriver Foundation is thanked for their generous support of our myxoid liposarcoma studies.

\section{Author details}

'Department of Pathology, The University of Texas MD Anderson Cancer Center, 1515 Holcombe Blvd Unit 085, Houston, TX 77030, USA. ${ }^{2}$ Sharette Institute of Oncology, Hadassah-Hebrew University Medical Center, POB 12000, Jerusalem 91120, Israel. ${ }^{3}$ Department of Medical Oncology, The University of Texas MD Anderson Cancer Center, 1515 Holcombe Blvd Unit

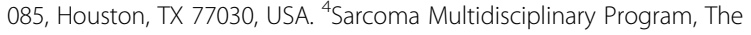
University Of Miami Sylvester Cancer Center, Room\#C-050, 1475 Northwest 12th Avenue, Suite 3513, Miami, FL 33136, USA. ${ }^{5}$ Department of Othropeadic Surgical Oncology, The University of Texas MD Anderson Cancer Center, 1515 Holcombe Blvd Unit 085, Houston, TX 77030, USA. ${ }^{6}$ Department of Radiation Oncology, The University of Texas MD Anderson Cancer Center, 1515 Holcombe Blvd Unit 085, Houston, TX 77030, USA. ${ }^{7}$ Department of Pathology, Texas Children's Hospital and Baylor College of Medicine, 6621 Fannin St, MC1-2261, Houston, TX 77030, USA. ${ }^{8}$ Department of Anatomic Pathology, General Hospital of Treviso, Piazza Ospedale 1, Treviso 31100, Italy. ${ }^{9}$ Department of Cancer Biology, The University of Texas MD Anderson Cancer Center, 1515 Holcombe Blvd Unit 085, Houston, TX 77030, USA.

${ }^{10}$ Sarcoma Research Center, The University of Texas MD Anderson Cancer Center, 1515 Holcombe Blvd Unit 085, Houston, TX 77030, USA.

${ }^{11}$ Department of Surgical Oncology, The University of Texas MD Anderson Cancer Center, 1515 Holcombe Blvd Unit 085, Houston, TX 77030, USA.

${ }^{12}$ Department of MusculoSkeletal Radiology, The University of Texas MD Anderson Cancer Center, 1515 Holcombe Blvd Unit 085, Houston, TX 77030, USA.

Received: 3 November 2012 Accepted: 20 December 2012 Published: 29 December 2012

\section{References}

1. Evans HL: Liposarcoma: a study of 55 cases with a reassessment of its classification. Am J Surg Pathol 1979, 3:507-523.

2. Kilpatrick SE, Doyon J, Choong PF, Sim FH, Nascimento AG: The clinicopathologic spectrum of myxoid and round cell liposarcoma. A study of 95 cases. Cancer 1996, 77:1450-1458.

3. Antonescu C, Ladanyi M: Myxoid Liposarcoma. In Pathology and genetics of Tumuors of bone and soft tissue. Edited by Fletcher CDM, Unni KK, Mertens F. Lyon: International Agency for Research on Cancer Press; 2002.

4. Weiss SW, Goldblum JR: Enzinger and Weiss's soft tissue tumors. $5^{\text {th }}$ edition. Maryland Heights, MO: Mosby Elsevier; 2008:498-510.

5. Haniball J, Sumathi VP, Kindblom LG, et al: Prognostic factors and metastatic patterns in primary myxoid/round-cell liposarcoma. Sarcoma 2011, 538085

6. Antonescu CR, Tschernyavsky SJ, Decuseara R, et al: Prognostic impact of P53 status, TLS-CHOP fusion transcript structure, and histological grade in myxoid liposarcoma: a molecular and clinicopathologic study of 82 cases. Clin Cancer Res 2001, 7:3977-3987.

7. Fritchie KJ, Goldblum JR, Tubbs RR, et al: The expanded histologic spectrum of myxoid liposarcoma with an emphasis on newly described patterns: implications for diagnosis on small biopsy specimens. Am J Clin Pathol 2012, 137:229-239.

8. Antonescu CR, Elahi A, Humphrey M, et al: Specificity of TLS-CHOP rearrangement for classic myxoid/round cell liposarcoma: absence in 
predominantly myxoid well-differentiated liposarcomas. J Mol Diagn 2000, 2:132-138.

9. Bode-Lesniewska B, Frigerio S, Exner U, Abdou MT, Moch H, Zimmermann DR: Relevance of translocation type in myxoid liposarcoma and identification of a novel EWSR1-DDIT3 fusion. Genes Chromosomes Cancer 2007, 46:961-971.

10. Crozat A, Aman P, Mandahl N, Ron D: Fusion of CHOP to a novel RNAbinding protein in human myxoid liposarcoma. Nature 1993, 363:640-644

11. Hisaoka M, Tsuji S, Morimitsu Y, et al: Detection of TLS/FUS-CHOP fusion transcripts in myxoid and round cell liposarcomas by nested reverse transcription-polymerase chain reaction using archival paraffin-embedded tissues. Diagn Mol Pathol 1998, 7:96-101.

12. Knight JC, Renwick PJ, Dal Cin P, Van den Berghe H, Fletcher CD: Translocation $\mathrm{t}(12 ; 16)(\mathrm{q} 13 ; \mathrm{p} 11)$ in myxoid liposarcoma and round cell liposarcoma: molecular and cytogenetic analysis. Cancer Res 1995, 55:24-27.

13. Panagopoulos I, Mandahl N, Ron D, et al: Characterization of the CHOP breakpoints and fusion transcripts in myxoid liposarcomas with the 12;16 translocation. Cancer Res 1994, 54:6500-6503.

14. Powers MP, Wang WL, Hernandez VS, et al: Detection of myxoid liposarcoma-associated FUS-DDIT3 rearrangement variants including a newly identified breakpoint using an optimized RT-PCR assay. Mod Pathol 2011, 23:1307-1315.

15. Charytonowicz E, Terry M, Coakley K, et al: PPARgamma agonists enhance ET-743-induced adipogenic differentiation in a transgenic mouse mode of myxoid round cell liposarcoma. J Clin Invest 2012, 122:886-898.

16. Cheng $H$, Dodge J, Mehl $E$, et al: Validation of immature adipogenic status and identification of prognostic biomarkers in myxoid liposarcoma using tissue microarrays. Hum Pathol 2009, 40:1244-1251.

17. Birch NC, Antonescu CR, Nelson M, et al: Inconspicuous insertion 22;12 in myxoid/round cell liposarcoma accompanied by the secondary structural abnormality der(16)t(1;16). J Mol Diagn 2003, 5:191-194.

18. Dal Cin P, Sciot R, Panagopoulos I, et al: Additional evidence of a variant translocation $\mathrm{t}(12 ; 22)$ with EWS/CHOP fusion in myxoid liposarcoma: clinicopathological features. J Pathol 1997, 182:437-441.

19. Hosaka T, Nakashima Y, Kusuzaki K, et al: A novel type of EWS-CHOP fusion gene in two cases of myxoid liposarcoma. J Mol Diagn 2002, 4:164-171.

20. Mrozek K, Szumigala J, Brooks JS, Crossland DM, Karakousis CP, Bloomfield CD: Round cell liposarcoma with the insertion (12;16)(q13;p11.2p13). Am J Clin Pathol 1997, 108:35-39.

21. Negri T, Virdis E, Brich $S$, et al: Functional mapping of receptor tyrosine kinases in myxoid liposarcoma. Clin Cancer Res 2010, 16:3581-3593.

22. Demicco EG, Torres KE, Ghadimi MP, et al: Involvement of the PI3K/Akt pathway in myxoid/round cell liposarcoma. Mod Pathol 2012, 25:212-221.

23. Barretina J, Taylor BS, Banerji S, et al: Subtype-specific genomic alterations define new targets for soft-tissue sarcoma therapy. Nat Genet 2010, 42:715-721.

24. Grosso F, Jones RL, Demetri GD, et al: Efficacy of trabectedin (ecteinascidin-743) in advanced pretreated myxoid liposarcomas: a retrospective study. Lancet Oncol 2007, 8:595-602.

25. Casali PG, Sanfilippo R, D'Incalci M: Trabectedin therapy for sarcomas. Curr Opin Oncol 2010, 22:342-346.

26. Grohar PJ, Griffin LB, Yeung C, et al: Ecteinascidin 743 interferes with the activity of EWS-FLI1 in Ewing sarcoma cells. Neoplasia 2011, 13:145-153.

27. Grosso F, Sanfilippo R, Virdis E, et al: Trabectedin in myxoid liposarcomas (MLS): a long-term analysis of a single-institution series. Ann Oncol 2009, 20:1439-1444

28. Demetri GD, Fletcher CD, Mueller $E$, et al: Induction of solid tumor differentiation by the peroxisome proliferator-activated receptor-gamma ligand troglitazone in patients with liposarcoma. Proc Natl Acad Sci U S A 1999, 96:3951-3956.

29. Roberge D, Skamene T, Nahal A, Turcotte RE, Powell T, Freeman C Radiological and pathological response following pre-operative radiotherapy for soft-tissue sarcoma. Radiother Oncol 2010, 97:404-407.

30. Downs-Kelly E, Goldblum JR, Patel RM, et al: The utility of fluorescence in situ hybridization (FISH) in the diagnosis of myxoid soft tissue neoplasms. Am J Surg Pathol 2008, 32:8-13.

31. Weaver J, Downs-Kelly E, Goldblum JR, et al: Fluorescence in situ hybridization for MDM2 gene amplification as a diagnostic tool in lipomatous neoplasms. Mod Pathol 2008, 21:943-949.
32. Gronchi A, Bui BN, Bonvalot $\mathrm{S}$, et al: Phase II clinical trial of neoadjuvant trabectedin in patients with advanced localized myxoid liposarcoma. Ann Oncol 2012, 23:771-776.

33. Forni C, Minuzzo M, Virdis E, et al: Trabectedin (ET-743) promotes differentiation in myxoid liposarcoma tumors. Mol Cancer Ther 2009, 8:449-457.

34. Gerwirtz D: A Critical Evaluation of the mechanisms of action proposed for the antitumor effects of the anthracycline antibiotics adriamycin and daunorubicin. Biochem Pharmacol 1999, 57:727-741.

35. Kerbusch $\mathrm{T}$, de Kraker J, Keizer HJ, et al: Clinical pharmacokinetics and pharmacodynamics of ifosfamide and its metabolites. Clin Pharmacakinet 2001, 40:41-62.

36. Gamba-Vitalo C, Blair OC, Titton TR, Lane PA, Carbone R, Sartorelli AC: Cytotoxicity and differentiating actions of adriamycin in WEH1-3B D + leukemia cells. Leukemia 1987, 1:188-197.

37. Fornari FA, Jarvis WD, Grant $S$, et al: Induction of differentiation and growth arrest associated with nascent (nonoliogsomal) DNA fragmentation and reduced c-myc expression in MCF-7 human breast tumor cells after continous exposure to a sublethal concentration of doxorubicin. Cell Growth \& Differentiation 1994, 5:723-733.

38. Smith LM, Anderson JR, Coffin CM: Cytodifferentiation and clinical outcome after chemotherapy and radiation therapy for rhabdomyosarcoma (RMS). Med Pediatr Oncol 2002, 38:398-404.

39. Huang HY, Antonescu CR: Molecular variability of TLS-CHOP structure shows no significant impact on the level of adipogenesis: a comparative ultrastructural and RT-PCR analysis of 14 cases of myxoid/round cell liposarcomas. Ultrastruct Pathol 2003, 27:217-226.

doi:10.1186/2045-3329-2-25

Cite this article as: Wang et al: Extensive adipocytic maturation can be seen in myxoid liposarcomas treated with neoadjuvant doxorubicin and ifosfamide and pre-operative radiation therapy. Clinical Sarcoma Research 2012 2:25.

\section{Submit your next manuscript to BioMed Central and take full advantage of:}

- Convenient online submission

- Thorough peer review

- No space constraints or color figure charges

- Immediate publication on acceptance

- Inclusion in PubMed, CAS, Scopus and Google Scholar

- Research which is freely available for redistribution 